Décadrages Décadrages

cinéma, à travers champs Cinéma, à travers champs

$14 \mid 2009$

Cinéma et migration

\title{
Españolas en París ou la solitude des « bonnes à tout faire » espagnoles à Paris
}

Bruno Tur

\section{(2) OpenEdition}

1 Journals

Édition électronique

URL : https://journals.openedition.org/decadrages/332

DOI : $10.4000 /$ decadrages.332

ISSN : 2297-5977

Éditeur

Association Décadrages

Édition imprimée

Date de publication : 1 avril 2009

Pagination : 66-78

ISBN : 978-2-9700582-9-8

ISSN : 2235-7823

Référence électronique

Bruno Tur, «Españolas en París ou la solitude des «bonnes à tout faire » espagnoles à Paris », Décadrages [En ligne], 14 | 2009, mis en ligne le 10 avril 2010, consulté le 03 avril 2022. URL : http:// journals.openedition.org/decadrages/332; DOI : https://doi.org/10.4000/decadrages.332 
1 Bruno Tur travaille au Centre de recherches ibériques et ibéro-américaines, à I'Université de Paris Ouest Nanterre La Défense.

2 Sur le contexte historique et l'analyse du discours, voir Isabel Alonso Dávila, "Españolas en París: una película, un relato de vida, un retrato de emigración femenina ", dans José Manuel Trujillano Sánchez et José María Gago González (éd.), Jornadas "Historia y fuentes orales". Historia y memoria del franquismo, 1936-1978, Fundación Cultural Santa Teresa, Avila, 1997, pp. 155-164.

3 Lors de sa sortie en Espagne, Españolas en París n'a pas eu de problèmes avec la censure. Toute fiction sur des Espagnols installés en France devait éveiller les soupçons des censeurs, puisque nous savons que la France fut le principal pays d'accueil des exilés politiques après la Guerre civile. C'est donc pour contourner tout problème que Bodegas a, par exemple, modifié le caractère de l'un de ses personnages: "Nous avons écrit un premier scénario que nous n'avons pas tourné parce qu'il aurait eu des problèmes avec la censure car l'un des personnages [...] était un déserteur. Finalement, on s'est mis d'accord pour lui donner un angle plus proche de la comédie" (El País, 28.08.82, notre traduction). Españolas en París ne fait jamais référence à la communauté des immigrés politiques espagnols à Paris, pourtant nombreux. Par ailleurs, puisqu'il évoque une rencontre amoureuse entre deux immigrés et insinue des relations sexuelles entre les deux célibataires, le film aurait pu être censuré. Mais paradoxalement, il collait au discours du franquisme sur l'émigration des femmes et sur les "dangers" auxquels elles allaient être confrontées, nous y reviendrons (cf. note 13).

4 José María Caparrós Lera, El cine español bajo el régimen de Franco, 1936-1975, Edicions Universitat de Barcelona, Barcelona, 1983, pp. 57-58.

5 Entretien avec Roberto Bodegas, abc guionistas, 08.05.05 (notre traduction).

6 Laura Oso Casas, Españolas en París. Estrategias de ahorro y consumo en las migraciones internacionales, Bellaterra, Barcelona, 2004.

7 Le scenario de Españolas a d'abord été écrit avec de Chalonge, puis avec José Luis Dibildos (producteur du film) et Antonio Mingote. L'un des tapuscrits est conservé à la Cinémathèque de Paris.

8 Le Journal du Dimanche, 13.02.72.
Españolas en París ou la solitude des "bonnes à tout faire " espagnoles à Paris par Bruno Tur 1

En 1971, le film Españolas en París (Roberto Bodegas) inaugurait la Tercera Vía [troisième voie] du cinéma espagnol2. Alors que le dictateur Franco n'était pas encore mort et que toute production était soumise à la censure ${ }^{3}$, deux courants s'étaient imposés dans la péninsule : un cinéma populaire et un autre plus intellectuel4. Le propos de la Tercera Vía - expression créée par la critique, mais dont Bodegas s'attribue parfois la paternité - fut de proposer un cinéma ni populaire ni intellectuel, mais plus proche des réalités sociales des Espagnols: "Il s'agissait de changer un cinéma franquiste où les personnages étaient en-dehors de la réalité sociale» $\mathbf{5}$. Dirigé par Roberto Bodegas et produit par José Luis Dibildos, Españolas en París fut aussi le film témoin de l'un des évènements historiques les plus marquants du franquisme : celui de l'émigration vers l'Europe de centaines de milliers d'Espagnols et, plus particulièrement, l'émigration de jeunes femmes parties s'employer dans les métiers domestiques de la bourgeoisie parisienne ${ }^{6}$.

Dans ce film, on suit le parcours d'Isabel (interprétée par Ana Belén), depuis son arrivée dans la capitale française et tout au long de son expérience de "bonne à tout faire» dans la capitale. Roberto Bodegas décortique la découverte de la ville, les relations entre immigrées, le premier flirt et les rapports avec les employeurs. Le réalisateur fut lui-même un immigré espagnol en France, à la fin des années 1950, travaillant d'abord dans un garage, avant de s'intéresser au cinéma et d'assister Christian de Chalonge dans la réalisation d'O Salto (1967), documentaire sur l'émigration portugaise 7 . Ces jeunes femmes espagnoles «bonnes à tout faire», Bodegas les a bien connues:

«Le sujet des Espagnoles à Paris [...], je l'avais là, en moi, dit Bodegas en se frappant la poitrine. Dès 1957, j’ai fréquenté ce milieu de l'émigration espagnole à Paris et ses rendez-vous de l'avenue de Wagram.»8

Ou encore : 
"J'ai toujours pensé que ma première réalisation porterait sur les bonnes espagnoles: c'était, pour moi, une sorte de dette d'honneur, un moyen de me délivrer d'un tas de choses. » 9

Si Españolas en París, qui est donc son premier film, est très largement inspiré de sa propre expérience, il n'en demeure pas moins une fiction qui doit nous interroger sur le regard porté par son auteur sur cette émigration, ainsi que sur la vie des femmes espagnoles à Paris. En effet, alors que la vision largement dominante présentait ces immigrées comme des filles simplettes venues gagner un peu d'argent pour rentrer rapidement en Espagne, Bodegas contourne cette représentation en construisant son film autour des rapports entre plusieurs immigrées aux personnalités bien distinctes et aux objectifs tout aussi différents $\mathbf{1 0}$. Bodegas fait également rapidement allusion aux histoires de papiers des immigrés (carte de séjour, carte de travail, passeport). A travers un de ses personnages (Manolo), il ira même jusqu'à émettre une critique sur le cloisonnement administratif qui empêche tout immigré en France d'exercer une autre profession que celle inscrite sur sa carte de travail11: dans une moindre mesure, il s'agit donc aussi d'un film engagé. D’ailleurs, c'est bien dans cette optique que Bodegas a créé le personnage d'Emilia, dont il dit qu'il

«s'agit d'une fille de la petite bourgeoisie à laquelle son instruction aurait permis d'être secrétaire par exemple; mais la difficulté d'un contrat de travail de ce genre pour une étrangère l'a amenée à devenir bonne. $\mathbf{1 2}^{\mathbf{2}}$

Le film a néanmoins la particularité de mettre l'accent sur une question qui préoccupait les familles restées au village: celle de la sexualité de ces jeunes femmes célibataires, et d'une possible maternité en dehors du mariage.

Dans Españolas en París, il n'y a pas un, mais plusieurs personnages principaux. Bien sûr, l'argument du film tourne autour de la vie d'Isabel, jeune immigrée dans la capitale française. Mais Roberto Bodegas a très clairement voulu montrer la diversité des profils présentés par l'immigration féminine espagnole.

\section{Les femmes au cœur de la fiction}

Isabel est jeune, elle a 19 ans. Elle est l'aînée de plusieurs frères et sœurs. C’est bien une caractéristique de l'émigration féminine espagnole, où l'aînée est la première à partir. L'argent envoyé à la famille restée sur place pouvait permettre de payer les études à l'un des garçons de la fratrie. C'est le cas d'Isabel qui, lors d'un échange avec sa patronne, puis en se confiant à son petit ami, parle de son frère et de l'intelligence de
9 "Entretien avec Roberto Bodegas", Les Nouvelles Littéraires, 14.02.72.

10 En Espagne comme en France, dans les années 1960 et jusqu'à nos jours, les immigrées espagnoles ont été perçues comme des jeunes filles rurales aux facultés intellectuelles limitées, très croyantes et attachées aux traditions locales, sachant à peine lire et écrire et, donc, incapables d'apprendre le français.

11 Dans les années 1960, les immigrés venus en France se voyaient remettre une carte de travail sur laquelle étaient stipulées leur profession et leur région de résidence. Ils ne pouvaient y exercer un autre métier ou travailler dans une autre région sans l'accord de l'administration, ce qui ne facilitait pas l'ascension professionnelle ou la mobilité géographique. Sur cet aspect de la politique française d'immigration, voir Alexis Spire, Etrangers à la carte. L'administration de l'immigration en France (19451975), Grasset, Paris, 2005.

12 "Entretien avec Roberto Bodegas", Les Nouvelles Littéraires, 14.02.72. Outre ce que nous avons déjà dit en note précédente, les Espagnoles se voyaient presque exclusivement autorisées à travailler dans les métiers des services. 
Emilia, se faisant passer pour une Française, séduit un cadre espagnol en voyage d'affaire à Paris

celui-ci (sous-entendue sa capacité à étudier). Sitôt arrivée à Paris, Isabel est placée et se met à travailler, alors qu'elle ne parle pas un mot de français. Ses employeurs n'ont pas d'enfants. Leur appartement, dans le $\mathrm{XVI}^{\mathrm{e}}$ arrondissement de la capitale, à Passy, est très grand. Mais on met à la disposition d'Isabel une chambre de bonne au sixième étage.

Elle y retrouve Emilia (Laura Valenzuela), sa voisine de chambre, plus âgée qu'elle, employée de maison également, qui trompe sa solitude en se faisant passer pour une Française et va cueillir, à la gare d'Austerlitz, les cuellos blancos, ces cadres espagnols venus travailler à Paris. Emilia maîtrise donc parfaitement le français pour pouvoir mener à bien cette mascarade. Femme libre refusant toute résignation à sa condition sociale, elle n'envisage absolument pas de retourner en Espagne et dans son village, où elle a une réputation sulfureuse. Elle se démarque des autres filles (émigrées économiques) et passerait presque, d'après les caractéristiques apposées aux uns et aux autres, pour une exilée politique. Emilia est une intellectuelle et une rebelle. Coiffée d'une perruque, elle se fait passer pour une femme bourgeoise française sans éveiller les soupçons de ses prétendants, pourtant Espagnols comme elle. Renvoyée par son employeuse après avoir introduit son amant dans l'appartement de celleci, elle devient toiletteuse pour chiens et semble quitter définitivement le métier d'employée de maison: au lieu de servir les dames, elle servira désormais leurs mascottes.

Francisca (Tina Sainz), quant à elle, croise le chemin d'Isabel alors qu'elles font les courses pour leur patronne. Francisca ne parle, ni ne comprend le français. Elle ne se plaît pas à Paris et souhaite repartir en Espagne, ce qu'elle fera d'ailleurs. Elle est l'opposé du personnage d'Emilia.

Isabel rencontre aussi Dioni (Elena Maria Tejero), dont l'objectif est d'économiser suffisamment pour se constituer une dot alors que

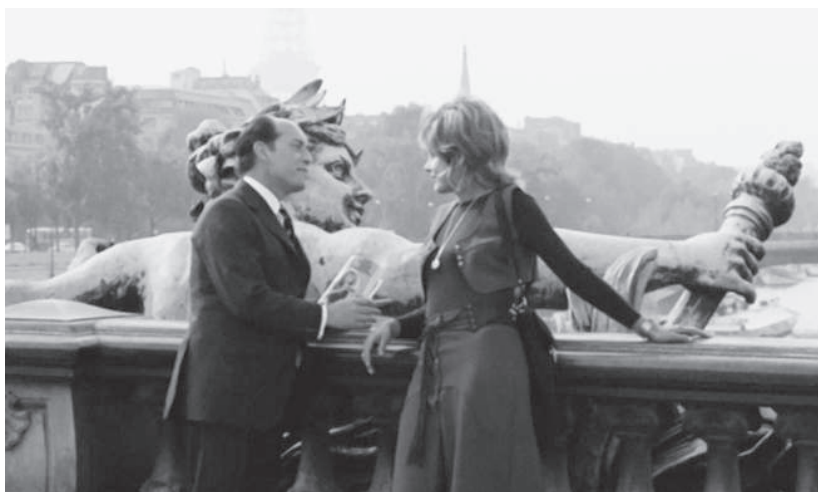


son fiancé, Antonio, travaille en Allemagne. Grâce aux émissions de radio diffusées sur ondes longues à destination des Espagnols émigrés en Europe, ils se dédicacent des chansons. Avant la fin du film, il viendra la chercher en voiture à Paris pour retourner en Espagne et s'y marier13. Mais avant de partir, Dioni aura fait venir d'Espagne sa jeune cousine Casilda, qui héritera de sa "place» et de sa chambre14.

Quatre femmes, quatre immigrées espagnoles à Paris dont les personnalités et les situations particulières dans la migration forment une palette de profils distincts. Bodegas souhaitait expressément faire un film sur les femmes émigrées, et c'est pourquoi l'immigration masculine est réduite aux personnages de Manolo (chauffeur de l'employeuse d'Emilia et séducteur d'Isabel) et, dans une moindre mesure, d'Antonio, le promis de Dioni. Ce n'est que lorsque Isabel annoncera à Manolo qu'elle est enceinte de lui qu'on verra un groupe d'hommes immigrés. Nous y reviendrons.

On comprend que Bodegas a créé chaque personnage pour rendre compte de la diversité de ces profils et pour rompre l'image convenue de l'immigrée espagnole très jeune, un peu perdue loin de son village. On trouve ainsi la nouvelle arrivée au futur incertain (Isabel), l'employée expérimentée et parfaitement à l'aise à Paris (Emilia), la femme rangée qui n'attend que son mariage (Dioni) et, enfin, l'immature qui ne peut s'épanouir à Paris et pour qui l'émigration est un échec (Francisca). Différentes générations, différents objectifs, différentes situations, c'est bien là l'une des caractéristiques du film: montrer que derrière chaque femme émigrée se cachait une histoire particulière, alors que le régime franquiste véhiculait une seule et même image de l'émigration féminine: celle d'une femme accompagnant le migrant, mais n'émigrant pas de son propre chef. Outre le fait que Franco jugeait publiquement ces départs inutiles, il mettait en garde «les jeunes filles» contre "les dangers d'une telle aventure»15. Constructive pour les hommes, l'émigration n'avait pas lieu d'être pour les femmes. Españolas en París est venu rappeler à l'opinion publique espagnole que l'émigration féminine était une réalité sociale $\mathbf{1 6}$.

\section{Les personnages non immigrés, de l'autre côté du miroir social}

Cette réalité sociale de ces Espagnoles, Bodegas réussit à la confronter à l'aisance économique de la classe élevée dans une scène où Isabel sert le dîner aux invités de ses employeurs, un couple d'Espagnols venus faire des affaires à Paris. Alors que l'épouse du négociant a sensiblement le même âge qu'Isabel, on mesure le fossé qui les sépare à travers un seul geste et quelques paroles: alors qu'Isabel tend sa main pour
13 Sur la place symbolique du transistor ou de la voiture dans l'émigration espagnole en France, voir Olga Muro Gil, "Logiques migratoires: les immigrés espagnols en France, 19551970 ", in Migrations Société, vol. 18, n 104, mars-avril 2006, pp. 41-66.

14 Bodegas illustre par cet exemple la construction des réseaux d'émigration féminins espagnols. De la même façon, Emilia et Isabel sont originaires du même village: c'est la première qui a trouvé la place de la deuxième, avant même son arrivée à Paris.

15 Francisco Franco Bahamonde, Discursos y mensajes del Jefe del Estado, 1964-1967, Dirección general de cultura popular y espectáculos, Publicaciones españolas, Madrid, 1968, p. 175. Le dictateur considérait que les femmes migrantes seraient confrontées à des dangers qu'elles ne rencontraient pas en Espagne: sexualité hors mariage, réseaux de prostitution, travail illégal, etc.

16 Bruno Tur, "L'émigration des femmes sous le franquisme: législation, discours officiels et stratégies personnelles dans les années 1960 ", dans Philippe Rygiel (éd.), Politique et administration du genre en migration, Editions Aux lieux d'être / CNHI, La Courneuve, à paraître en 2009. 
17 La rue de la Pompe, dans le XVle arrondissement, l'un des plus cossus de la capitale, comptait beaucoup d'Espagnoles venues s'employer dans les familles du quartier. On y trouve encore l'église espagnole, toujours en activité. Le fait de baptiser Pompe Service les cours pour employeurs est donc, pour Bodegas, un clin d'œil à ce quartier. Voir Isabelle TaboadaLeonetti, Les immigrés des beaux quartiers. La communauté espagnole du XVI ${ }^{e}$, L'Harmattan, Paris, 1987.

18 Les syndicats CGT, CFDT ou encore celui de la Jeunesse Ouvrière Chrétienne Féminine (JOCF), organisèrent par exemple dès les années 1960 des groupes de discussions avec, d'un côté, des travailleuses employées de maison et, de l'autre, des employeuses. II s'agissait de recueillir des témoignages sur la vie des employés domestiques, particulièrement lorsqu'ils étaient immigrés, pour connaître leurs situations et coller à leurs revendications, le but étant d'intégrer les travailleurs étrangers dans les syndicats.

19 "Entretien avec Roberto Bodegas ", Les Nouvelles Littéraires, 14.02.72. serrer celle de l'invitée, celle-ci ne bouge pas et se contente de la fixer du regard, puis de l'interroger sur ses origines en la tutoyant. S'adressant à l'employeuse, après avoir appris le montant du salaire versé à Isabel, sa conclusion sera la suivante: "Vous nous les pourrissez avec ce que vous leur donnez!»

Cependant, les Lemonier, employeurs d'Isabel, ne portent pas le même regard sur elle. Ils semblent être à la fois mal à l'aise et affectueux vis-à-vis de leur "bonne». Dès le début, un fort rapport hiérarchique s'instaure entre les deux femmes. L'homme est en retrait, s'adressant à Isabel de façon infantilisante, mais toutefois gentille et, finalement, un peu stupide. La femme est parfois patiente, parfois agacée quand Isabel ne comprend pas une demande ou ne respecte pas une recette. Malgré une bonne volonté des deux côtés, force est de constater que c'est l'incompréhension qui domine. Une incompréhension qui s'explique par deux facteurs principalement: d'une part, la langue, puisque ni les immigrées ne parlent le français à leur arrivée, ni les employeuses ne maîtrisent l'espagnol; d'autre part, la différence des coutumes et des modes de vies espagnols et français expliquent aussi cette incompréhension: en arrivant dans les cuisines cossues des beaux quartiers parisiens, les jeunes espagnoles découvraient les robots électroménagers, la façon de conserver un fromage ou même des produits comme le pamplemousse, que certaines n'avaient jamais vu auparavant. La vie rurale espagnole des années 1960 était à mille lieues de la vie parisienne: mœurs et techniques d'origine sont à mettre de côté par l'immigrée, puisque c'est elle qui doit s'adapter aux appartements parisiens. Cependant, nous ne sommes pas surpris de voir l'employeuse d'Isabel fréquenter les cours de Pompe Service ${ }^{\mathbf{1 7}}$, où on lui reproche de ne pas s'intéresser à la vie d'Isabel - tant à sa vie en Espagne qu'à sa situation en France. Ces types de cours, qui ont bien existé, témoignent de la sensibilité d'une certaine catégorie d'employeuses vis-à-vis de leurs employées. On trouvait, à la base de ces groupes, des syndicats $\mathbf{1 8}$ et des mouvements religieux. Ils furent bien sûr utiles aux employeuses, mais peu fréquentés dans l'ensemble.

Quoi qu'il en soit, Bodegas ne dresse pas un portrait sévère des employeurs. Au contraire, Madame Lemonier (Françoise Arnoult) apparaît comme une femme sympathique et avenante, mais qui garde ses distances avec son employée, d'abord mise en confiance: "[Les patrons], tout en étant assez gentils, tombent plutôt dans la démagogie, le paternalisme, cultivant les idées toutes faites et ne comprenant rien à cet autre monde »19, dira le réalisateur. Ainsi, lorsqu'Isabel annonce sa grossesse à sa patronne alors que celle-ci s'apprête à partir en week-end : "Ce n'est pas grave Isabel, c'est des choses qui arrivent. On verra tout ça à notre 
retour», puis elle est interrompue par son époux: "Dépêche-toi, on va tomber dans les embouteillages!» $\mathbf{2 0}$.

Enfin, parmi les personnages secondaires, on trouve la guichetière chargée d'effectuer un virement pour l'Espagne et qui s'exaspère de ne pas être comprise par les immigrées; il y a aussi l'employé du consulat ou le médecin du travail qui annonce à Isabel, dans la froideur de son cabinet, mais sur un ton bienveillant, qu'elle est enceinte. Le plus important de ces personnages secondaires est celui de la concierge, qu'on ne voit qu'au début du film, quand Isabel, accompagnée par Emilia venue la chercher à la descente du car qui l'a amenée d'Espagne, entre pour la première fois dans le hall de l'immeuble dans lequel elle va vivre désormais. Après avoir rappelé à Emilia que les voisins s'étaient plaints du transistor qu'elle écoute pendant la nuit, la concierge regarde Isabel s'engouffrer dans l'escalier de service et exprime sa pensée à voix haute, l'air dépité : «Encore une autre...». Cette séquence illustre l'opposition qui, d'après les témoignages que nous avons recueillis au cours de nos recherches, caractérisait les relations entre les concierges et les habitantes espagnoles (puis portugaises) du sixième étage, où se situaient les chambres de bonnes $\mathbf{2 1}$.

\section{Des personnages en quête de compagnie}

Le point commun entre tous ces personnages n'est jamais clairement énoncé, mais saute aux yeux du spectateur. Il apparaît dès les premières scènes et traverse tout le film comme s'il était le fil qui le tient, accentué ici par la musique aux notes angoissantes, là par des plans serrés sur quelques visages. Ce point commun, c'est la solitude. La solitude d'Isabel, qui laisse famille et amis en Espagne pour vivre "l'aventure " migratoire. La solitude d'Emilia, qu'elle essaie de combler en séduisant des touristes espagnols et en imitant «les habitudes, les manières de la bourgeoisie française dont elle n'est qu'un reflet» $\mathbf{2 2}$; celle de Dioni, dont le futur mari se trouve en Allemagne depuis cinq ans; celle de Francisca dans une ville, Paris, qui ne lui ressemble pas. Mais c'est aussi la solitude de Madame Lemonier, femme au foyer dans un appartement trop grand pour elle; celle de l'invitée espagnole, dont on comprend bien que son époux ne lui est pas fidèle. Et c'est la solitude de Manolo, enfin, qui garde dans son portefeuille la photo de sa fiancée restée en Espagne, tout en embrassant Isabel.

Toute la finesse du film, son ciment, tient dans ce sentiment qui, paradoxalement, lie les personnages entre eux. Plusieurs scènes l'illustrent particulièrement: par exemple, un soir où Dioni entend Isabel et Manolo faire l'amour dans la chambre voisine, elle est prise d'un
20 Pour Isabel, la grossesse et l'enfant à naître posent avant tout un problème moral. Madame Lemonier, quant à elle, ne considère que l'aspect matériel.

21 Cette opposition s'explique d'abord par le fait que la concierge (souvent française à cette époque) est garante de la tranquillité de l'immeuble et des propriétaires/locataires: le sixième est donc un endroit placé sous surveillance; mais aussi pour des raisons professionnelles, puisque les Espagnoles, qui avaient compris l'intérêt d'une loge pour travailler, économiser et élever leurs enfants en même temps, visaient ces places de concierges quelques années après leur arrivée.

22 "Entretien avec Roberto Bodegas ", Les Nouvelles Littéraires, 14.02.72. 
23 Télérama, 12.02.72.

24 Voir Pierre Nora (dir.), Les lieux de mémoire, Gallimard (Quarto), Paris, 1997, 3 tomes.

25 Le Figaro, 17.02.72.

26 Bruno Tur, "L'immigration espagnole dans la France des années 1960-1970: sources iconographiques et épistolaires dans les collections privées", in Regards, n 13, hiver 2009 (à paraître).

27 Nous avons rencontré des propos similaires au cours de nos entretiens avec des migrants espagnols: "Tu te rends compte, avec un seul ticket de métro, tu peux visiter toute la ville par en dessous". (Entretien avec Encarna Molla, immigrée valencienne retraitée, Paris, 13 septembre 2005). désir sexuel qu'elle ne peut assouvir puisque Antonio est absent. Autre exemple : après que le médecin a annoncé à Isabel qu'elle allait avoir un enfant, c'est seule qu'elle quitte le cabinet et traverse la salle d'attente, où d'autres travailleuses espagnoles la regardent passer sans la voir. Elle sera tout aussi seule lorsque Manolo disparaîtra quelques jours, après qu'elle lui a annoncé qu'il allait être père. Cependant, elle le sera moins lorsqu'elle aura décidé de garder son enfant et que la fin du film la montrera le tenant dans ses bras. Quoiqu'il en soit, tout au long du film, Isabel est «seule, seule, comme ses amies espagnoles. Seule comme Francisca qui a le mal du pays. Seule comme Dioni qui économise sou à sou avant de rejoindre son fiancé. Seule comme Emilia qui erre dans Paris à la recherche de chaleur humaine. $\mathbf{2 3}$.

\section{Géographie parisienne de l'immigration espagnole}

Enfin, le film dessine une géographie parisienne de l'immigration espagnole, constituée de lieux de mémoire, tels que défini par Pierre Nora 24. Il s'agit d'abord de lieux matériels, à commencer par les sites les plus touristiques de la capitale: "On ne cesse guère d'accompagner la jeune héroïne au cours de ses promenades à travers la ville [...].» 25 Ainsi, on voit défiler l'Arc de Triomphe, près duquel se trouve le terminus de la ligne Iberbus reliant Paris à Valence, en Espagne. A certains moments, on devine le parvis de l'Opéra, les Galeries Lafayette, la Butte Montmartre et le Sacré Cœur, mais aussi la Tour Eiffel, plus visible: ce sont là les lieux les plus représentés lorsque l'on consulte les photographies conservées par les employées de maison $\mathbf{2 6}$. La scène la plus surprenante reste cependant celle où Francisca emmène Isabel découvrir Paris: elles ne sortiront pas du métro! Se rendant jusqu'à la station Louvre, qui dessert le musée du même nom, et qui est décorée par des moulures de statues religieuses, les deux «touristes " s'arrêtent devant la représentation d'une vierge, et engagent cette conversation:

Isabel: Quelle beauté! On dirait une église.

Francisca: je viens ici tous les dimanches. C'est la plus belle station de métro. [...] Pas vrai que le métro est magnifique? [...]

Isabel: Et au-dessus de nos têtes [à l'extérieur], qu'est-ce qu'il y a?

Francisca: Je ne sais pas. Au-dessus, il doit y avoir un de ces bordels!

Cet échange $\mathbf{2 7}$ illustre parfaitement le manque d'informations dont disposaient les émigrants espagnols sur la ville de destination. En général, ils découvraient la capitale en un jour, le dimanche suivant leur arrivée. Le reste du temps, ils se consacraient à leur travail et à la visite d'amis pendant leur temps libre. Si personne ne prenait le temps de 
leur montrer la capitale, ou s'ils n'étaient pas de nature curieuse, ils repartaient parfois de Paris sans en avoir vu les principaux monuments. Par ailleurs, puisqu'ils venaient de petits villages espagnols, ils pouvaient être impressionnés par le réseau souterrain du métro parisien, qui couvrait déjà pratiquement toute la capitale. Mais par-dessus tout, on comprend ici que Paris, destination principale des migrants espagnols en Europe, était souvent une ville inconnue dont on ne soupçonnait pas forcément la richesse patrimoniale: les jeunes migrants, éduqués dans les écoles de la Phalange franquiste, n'étaient pas initiés à l'importance du patrimoine étranger.

On voit d'autres lieux caractéristiques de l'immigration espagnole à Paris: l'intérieur du Consulat, sur le boulevard Malesherbes; une agence bancaire, pour envoyer l'argent épargné à la famille demeurée en Espagne. On s'étonne que d'autres lieux soient absents, mais on sait que le film, réduit dans sa durée, ne pouvait tous les contenir: l'église espagnole de la Rue de la Pompe, la promenade de l'avenue des Ternes n’apparaissent pas. La salle Wagram où les Espagnols aimaient danser le dimanche après-midi, et que Bodegas fréquentait par ailleurs, est évoquée une fois par Emilia, comme si le réalisateur avait refusé que ce lieu emblématique soit absent $\mathbf{2 8}$.

Basculant dans le domaine du privé, on trouve bien sûr l'appartement et, particulièrement, la cuisine 29. C'est là qu'Isabel passe la plus grande partie de ses journées. C’est là aussi, sur la table qui trône en son centre, qu'aura lieu à la fin du film la tentative d'avortement sur Isabel. Grande, moderne, spacieuse, cette cuisine contraste avec les chambres de bonnes exiguës et vieillottes où logent les domestiques. Celles-ci sont pourtant les lieux matériels les plus emblématiques de l'immigration féminine espagnole à Paris. Laura Oso a montré la fonction stratégique de ces

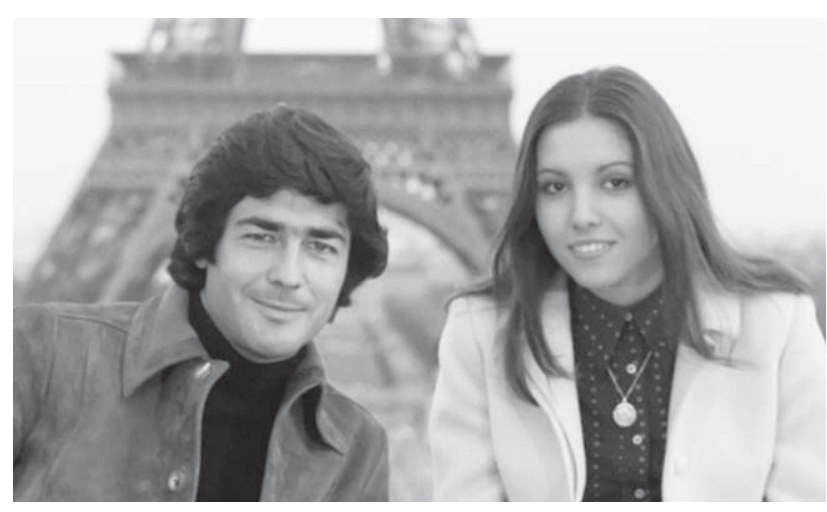

28 Sur tous ces lieux de rencontre, voir Bruno Tur, "Vie de couple et stratégies professionnelles des Espagnoles à Paris", in Hommes \& Migrations, $n^{\circ} 1262$, juillet-août 2006, pp. 3138.

29 Précisons que toutes les scènes d'intérieur ont été tournées en studio, à Madrid. Les scènes extérieures, tournées à Paris, n'ont fait l'objet d'aucune autorisation officielle, ce qui accentue l'aspect documentaire de certaines scènes. Voir le témoignage de Bodegas, El País, 28.08.82. 
30 Laura Oso Casas, "Chambras, loges, pubelas et burones: stratégies de mobilité sociale des Espagnoles à Paris", in Gabriel Gaso et Bruno Tur (dir.), Un siècle d'immigration espagnole en France, in Migrance hors-série, 2007, pp. 46-56.

31 Bruno Tur, "Sous les toits de Paris, les chambres de bonnes. Contribution à une histoire des lieux de mémoire de l'immigration espagnole en France ", in Regards, n 12, à paraître en 2009.

32 Natacha Lillo, La Petite Espagne de la PlaineSaint-Denis, 1900-1980, Autrement, Paris, 2004, pp. 61-62.

33 Cet extrait, qui peut surprendre aujourd'hui, a sûrement été inspiré à Bodegas par les nombreuses publications et émissions audiovisuelles des années 1960 sur les Espagnoles, comme le manuel de Solange Fasquelle, Conchita et Vous (1968). Sur toutes ces questions, voir Bruno Tur, "Représentations et stéréotypes sur l'immigration espagnole en France ", in Gabriel Gaso et Bruno Tur (dir.), Un siècle d'immigration espagnole en France, in Migrance hors-série, 2007, pp. 69-78. chambres mises à disposition, qui permettaient un hébergement gratuit sur le lieu de travail et, donc, une épargne importante ${ }^{30}$. La chambre de bonne est avant tout l'espace privé de l'employée. C'est là qu'elle se retrouve après une longue journée de travail; la chambre contient toutes ses affaires, toute sa vie parisienne, dirions-nous. Espace du privé donc, mais aussi espace de solitude, nous y revenons. Car l'employée s'y retrouve seule après le travail, et les témoignages recueillis vont dans ce sens 31 .

Mais les lieux de mémoires de cette immigration sont aussi immatériels, et nous en retrouvons certains dans ce film. C'est d'abord tout ce qui est lié à la langue, au français que les nouvelles arrivées découvraient et apprenaient difficilement, le parlant mal, inventant des mots constituant le fragnol, ce mélange d'espagnol et de français si caractéristique des différentes vagues migratoires espagnoles vers la France $\mathbf{3 2}$ : «tenemos las chambres» [nous avons les chambres], dira Emilia au début du film. Cette difficulté à parler le français était souvent soulevée par les humoristes de l'époque, et l'exemple le plus illustré était la soi-disant incapacité des Espagnoles à répondre correctement au téléphone. On le retrouve dans Españolas en París, lorsque son employeuse apprend à Isabel ce qu'elle doit dire lorsqu'elle est absente. D'ailleurs, à la fin, pour marquer l'adaptation réussie d'Isabel à la vie parisienne, on la verra répondre au téléphone de façon très satisfaisante. Le film joue ainsi avec ce stéréotype, qu'il reproduit donc pour finir par le contredire.

Autre stéréotype, celui de Conchita: c'est ainsi que l'employeuse nomme sa bonne fictive lors d'un cours de Pompe Service. D'ailleurs, l'assistante du formateur dresse un portrait sans équivoque des «bonnes» espagnoles:

«Les bonnes espagnoles sont fondamentalement travailleuses, mais mal organisées; joyeuses mais scandaleuses, elles et leurs transistors qu'elles mettent à plein volume; honnêtes mais très têtues; elles aiment beaucoup les enfants, mais elles reçoivent trop de visites familiales et amicales; sentimentales, mais capricieuses et arbitraires, avec des réactions incompréhensibles que certaines personnes interrogées estiment être d'une possible origine arabe.»33

\section{L'interrogation sur la sexualité des jeunes femmes émigrées}

Outre la solitude, c'est aussi la découverte d'une sexualité «libre» que le film veut narrer. Dans les campagnes espagnoles des années 1960, en plein franquisme, le contrôle social des adultes sur les jeunes filles était très important. Toute sexualité hors mariage était inenvisageable pour qui voulait conserver une bonne réputation au village. Ce n'est certes pas 
l'apanage du régime franquiste, mais ce contrôle était néanmoins institutionnalisé à travers des structures comme le service social des femmes (Auxilio Social) et la Phalange féminine, sans parler du poids de l'Eglise. En émigrant seules, ces jeunes filles échappaient à ce contrôle social. Au village, les absentes étaient parfois soupçonnées d'avoir une vie sexuelle peu conforme aux mœurs. Ces soupçons étaient prolongés par des rumeurs qui disaient que telle femme était enceinte d'un inconnu. Lorsque l'intéressée démentait ces dires, on pensait alors qu'elle avait peutêtre eu recours à un avortement. Ces rumeurs, nombreuses jusque dans les années 1980, ne se vérifient que très rarement dans les faits. Mais les quelques cas suffisaient à faire de toutes les émigrées des "putangues» et des avortées probables ${ }^{34}$.

C'est autour de ce thème que Bodegas a construit l'intrigue du film. Arrivée célibataire à Paris, Isabel rencontre Manolo, qui la séduit et dont elle tombe amoureuse. Mais Manolo a une fiancée à Madrid, qu'il doit épouser lorsqu'il aura économisé assez d'argent. Isabel le sait, mais elle est convaincue qu'il la choisira et quittera sa promise. Plusieurs scènes suggèrent des relations sexuelles entre les deux, ce qui se confirme lorsqu'Isabel apprend qu'elle est enceinte. Elle l'annonce à Manolo en le retrouvant dans un bar où celui-ci écoute un match de football à la radio avec des amis; il s'agit d'un groupe d'hommes immigrés espagnols, la seule fois du film où on les voit, comme si le réalisateur avait voulu stigmatiser l'ensemble des hommes migrants ${ }^{35}$. Manolo disparait les jours suivants, puis il revient pour retrouver Isabel. Enfin, il organise l'avortement en faisant appel à deux vieilles femmes espagnoles vêtues de noir. L'avortement était, bien sûr, un acte illégal. Mais au dernier moment, Isabel le refuse, et expulse les avorteuses, ainsi que Manolo, en criant sa colère: elle gardera l'enfant.

Les allusions au "qu'en dira-t-on" au village sont nombreuses dans le film. Par exemple, on sait qu'Emilia jouit au village d'une très mauvaise réputation, parce qu'elle a eu une liaison avec un homme marié. Par ailleurs, en s'adressant à Isabel pour la convaincre, l'une des avorteuses lui demande: "Tu veux rentrer au village avec un enfant dans les bras? Ils vont te faire un bon accueil!». D'ailleurs, c'est cette scène de la tentative d'avortement qui a été reprise dans l'une des trois affiches réalisées pour la sortie du film. Les deux premières ont peu d'intérêt. On y voit des visages de femmes se détachant sur un plan du métro parisien. Mais celle qui retient notre attention mérite une analyse plus détaillée.

On y reconnaît la silhouette d'Isabel, bien qu'elle porte une robe extrêmement courte d'un jaune fluorescent, robe dans laquelle elle n'apparaît jamais dans le film (où on la voit bien avec un ciré de cette couleur,
34 Bruno Tur, "Femmes séduites et forcément enceintes: la sexualité des immigrées espagnoles sous le regard des villages d'origine", in Migrance, $n^{\circ} 27$, mai 2007, pp. 79-85.

35 "En interrogeant ces filles, j'ai constaté qu'elles étaient plus souvent exploitées par leurs compatriotes que par leurs patrons", "Entretien avec Roberto Bodegas", Les Nouvelles Littéraires, 14.02.72. 
Affiche originale du film pour sa sortie en Espagne, 1971

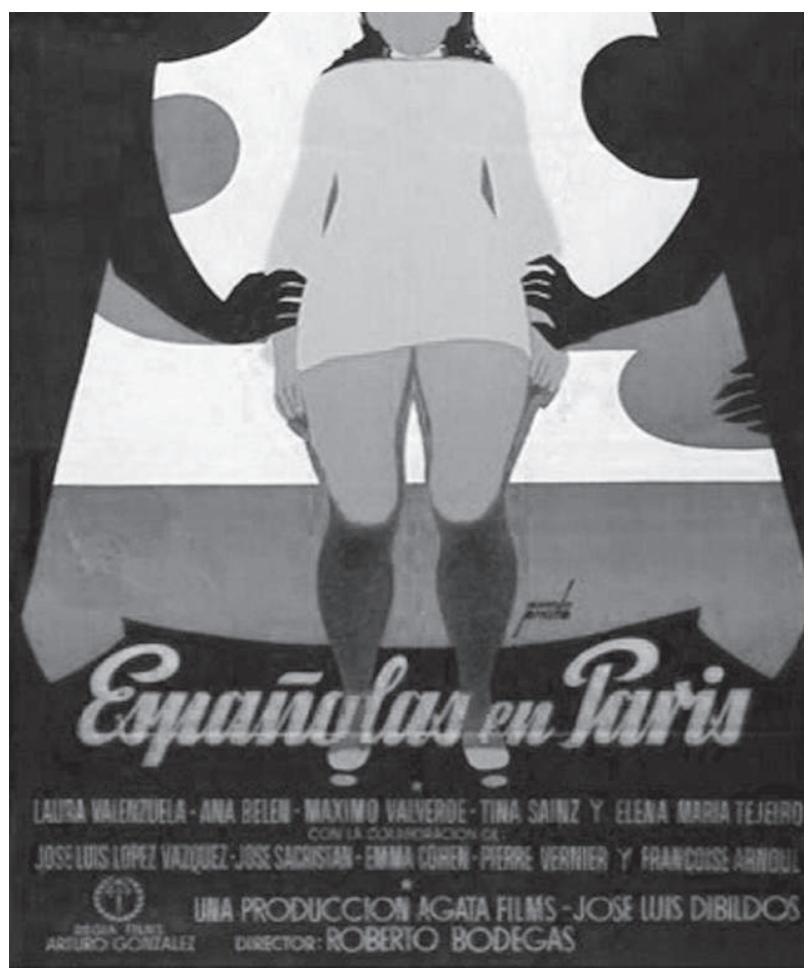

mais plus long). Il s'agit donc d'une liberté du dessinateur - dont on ne sait rien -, qui a voulu créer l'aspect provocateur de la jeune femme. Sur l'affiche, elle apparaît donc dans cette tenue, allongée sur une table d'un blanc immaculé, les jambes tombant dans le vide. Elle n'a pas de visage, c'est-à-dire pas d'yeux, pas de nez, pas de bouche : c'est Isabel, et c'est à la fois toutes les Espagnoles à Paris, comme le suggère le titre du film, à ses pieds, qui prend les allures d'une légende. Deux silhouettes noires sont penchées sur elle. On pourrait penser qu'il s'agit des deux avorteuses, mais ces silhouettes sont masculines. Leurs doigts pointus agrippent la victime. Cette affiche, censée représenter l'avortement à la fin du film, ressemble plus à une scène de viol sur le point d'être accompli. Dans sa robe fluorescente et très courte, Isabel apparaît comme une fille trop libre au goût du dessinateur. Ainsi, l'affiche se lit de cette façon: les Espagnoles à Paris sont trop modernes et deviennent des proies faciles d'hommes prêts à profiter de leur «innocence » $\mathbf{3 6}$.

A travers l'histoire d'Isabel et de ses amies, Roberto Bodegas a donc voulu rendre hommage à toute cette génération de femmes migrantes que l'Espagne de Franco, mais aussi la démocratie espagnole jusqu'à ces
36 C'est cette affiche qui a été reprise pour l'édition en DVD, dans une version retravaillée, en 2004. 
dernières années, a ignorées. Tourné en 1970, sorti en Espagne l'année suivante puis, en France, en 1972, ce film croise l'aspect documentaire et la construction dramatique pour offrir un film plus intéressant pour l'innovation de son approche sociale et des thèmes abordés que pour son histoire elle-même.

En France comme en Espagne, le film a bénéficié, lors de sa sortie, d'un accueil honorable de la critique. En Espagne, il aurait fait plus d'un million d'entrées. En France, le succès a été plus mitigé. L'accueil réservé par les «bonnes» espagnoles elles-mêmes en dit long sur les différentes façons de lire ce film ${ }^{\mathbf{3 7}}$.

En acceptant de garder son enfant, Isabel endosse le rôle des pionnières qui, depuis l'étranger, sans le savoir, ont souvent contribué à changer les mentalités en Espagne, à leurs dépens. Outre l'envoi des devises qui ont permis à l'Espagne de connaitre le développement que l'on sait, c'est peut-être le principal apport, pourtant méconnu, des émigrantes espagnoles: celui d'avoir bousculé les règles du jeu pour vivre leurs vies
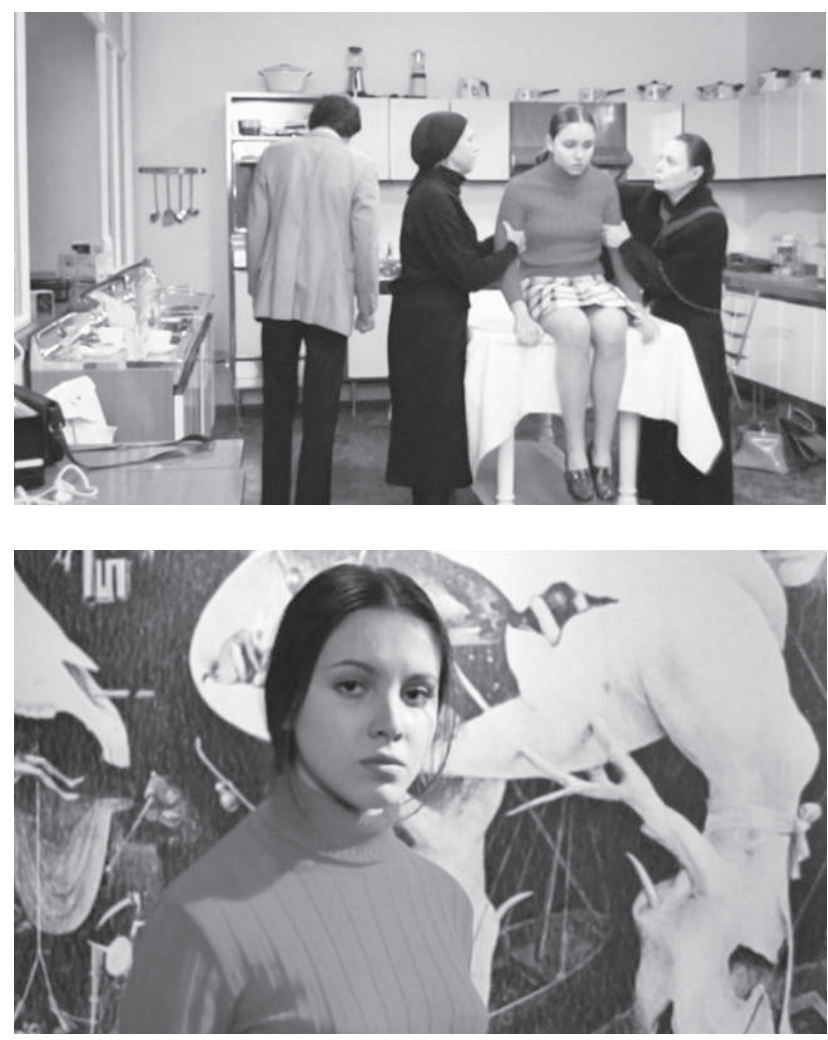

Dans la cuisine, au moment où

l'avortement va être pratiqué sur Isabel

37 Voir La Croix, 09.02.72.

Isabel avec, derrière elle, Le jardin des délices 
de femmes, loin du paternalisme franquiste et des rumeurs du village. Le personnage d'Emilia, les doutes de Dioni au moment de repartir, le cri de rage d'Isabel rendent bien compte de ce choix.

Clin-d'œil ou coïncidence: lorsque le film se termine sur un plan d'Isabel fixant la caméra après avoir répondu au téléphone, on peut voir à l'arrière une reproduction extraite du Jardin des délices, de Jérôme Bosch. Mais il s'agit du troisième panneau du triptyque: L'Enfer. Peutêtre le village, loin derrière elle...

\section{Españolas en París (1971)}

Scénario: Roberto Bodegas, Christian de Chalonge, José Luis Dibildos, Antonio Mingote. Réalisation: Roberto Bodegas. Image: Rafael de Casenave. Son: José María San Mateo. Montage: Petra de Nieva. Décors: Ramiro Gómez. Musique: Carmelo Bernaola. Production: José Luis Dibildos. Interprétation: Laura Valenzuela (Emilia), Ana Belén (Isabel), José Sacristán (Plácido), Tina Sáinz (Francisca), Máximo Valverde (Manolo), Elena María Tejeiro (Dioni), José Luis López Vázquez (Fernando), Emma Cohen (Katy), Pierre Vernier (M. Lemonier), Françoise Arnoul ( $\mathrm{M}^{\mathrm{me}}$ Lemonier), Teresa Rabal (Casilda) 